\title{
UTILIZAÇÃO DE LODO DE ESGOTO NA PRODUÇÃO DE MUDAS DE AROEIRA-PIMENTEIRA ${ }^{1}$
}

\author{
Rodrigo de Menezes Trigueiro² e Iraê Amaral Guerrini ${ }^{3}$
}

\begin{abstract}
RESUMO - O objetivo deste trabalho foi avaliar a viabilidade do uso de lodo de esgoto como componente do substrato para produção de mudas de aroeira-pimenteira (Schinus terebinthifolius Raddi). Para tanto, foram testadas as seguintes proporções de lodo de esgoto/casca de arroz carbonizada, em tubetes com $50 \mathrm{~cm}^{3}: 80$ / $20,70 / 30,60 / 40,50 / 50$ e 40/60, as quais foram comparadas ao substrato comercial comumente usado em viveiro florestal. Foram avaliados a altura de planta, o diâmetro de colo e o acúmulo de matéria seca de parte aérea e raiz, bem como se procedeu à análise química do tecido vegetal de parte aérea e raiz. As mudas de Schinus terebinthifolius desenvolvidas em substrato com 40, 50 e $60 \%$ de lodo de esgoto apresentaram crescimento inferior ao da testemunha, entretanto, considerando os parâmetros relação altura da planta/diâmetro do colo, produção de matéria seca de parte aérea e raiz e aspectos nutricionais, que atestam a qualidade da muda, os resultados foram satisfatórios. Portanto, conclui-se que o uso do resíduo lodo de esgoto na composição de substratos para a produção de mudas de espécies florestais é uma alternativa promissora.
\end{abstract}

Palavras-chave: Resíduos; Substrato; Espécies Florestais.

\section{USE OF SEWAGE SLUDGE FOR SEEDLINGS PRODUCTION OF BRAZILIAN PEPPER}

\begin{abstract}
The objective of this study was to evaluate the feasibility of the sewage sludge used as substrate component for seedlings production of Brazilian pepper (Schinus terebinthifolius Raddi). The following proportions of sewage sludge/carbonized rice hull were tested, in tubes with $50 \mathrm{~cm}^{3}: 80 / 20,70 / 30,60 / 40,50 / 50$ and $40 / 60$, which were compared to a commercial substrate commonly used by forest nursery. Growth seedlingsrelated parameters were assessed: shoot height, collar diameter and accumulation of dry matter on the shoot and root, chemical analysis of shoot and root tissues. Schinus terebinthifolius seedlings developed in substrate with 40, 50 e 60\% sewage sludge presented inferior growth compared to the control, however considering the parameters shoot height/collar diameter relation, production of dry matter on the shoot and root, and nutritional aspects, that attest to the quality of the seedlings, the results were satisfactory. Therefore, it was concluded that the use of this sewage sludge residue in the substrate composition for seedlings production of forest species is quite promising.
\end{abstract}

Keywords: Residues; Substrate; Forest species.

\footnotetext{
${ }^{1}$ Recebido em 02.12.2010 aceito para publicação em 05.06.2014

${ }^{2}$ Universidade Norte do Paraná, UNOPAR, Brasil. E-mail <r.trigueiro@hotmail.com.br>.

${ }^{3}$ Universidade Estadual Paulista, Fac. de Ciências Agronômicas, Campus de Botucatu, Brasil. E-mail: <iguerrini@fca.unesp.br>
} 


\section{INTRODUÇÃO}

A aroeira-pimenteira (Schinus terebinthifolius Raddi), pertencente à família Anacardiaceae, é uma espécie pioneira, nativa do Brasil. Tem potencial como ornamental, mas sua grande importância está na plasticidade ecológica, ou seja, em sua capacidade de se adaptar a uma ampla gama de ambientes, crescimento rápido e produção de frutos para a avifauna, o que a torna prioritária para a recuperação de áreas degradadas, especialmente matas ciliares (DURIGAN et al., 1997).

A preocupação mundial com a qualidade do meio ambiente faz que ocorra aumento na demanda de serviços e produtos florestais, em especial na produção de mudas para a recuperação de áreas degradadas, revegetação, reflorestamentos para fins econômicos, restauração de matas ciliares e arborização, entre outros fins (LELES et al., 2006). Em geral, áreas degradadas apresentam condições de crescimento limitantes para as espécies a serem introduzidas, como solos com baixa fertilidade e capacidade de retenção de água. Após o plantio no campo, um dos fatores na determinação da sobrevivência de uma espécie é seu desenvolvimento inicial na fase de muda, de modo que a habilidade competitiva e o uso de recursos por cada muda podem determinar a composição de espécies de árvores adultas em florestas (CROCE et al., 2007). A produção de mudas florestais, em quantidade e qualidade, é uma das fases mais importantes para o estabelecimento de bons povoamentos florestais com espécies nativas.

A boa formação de mudas destinadas à implantação de povoamentos mistos para fins de preservação ambiental e, ou, recuperação de áreas degradadas está relacionada a uma série de fatores, entre os quais se destaca a eficiência dos substratos utilizados. Na escolha de um substrato, devem-se observar, principalmente, suas características físicas e químicas, além dos aspectos econômicos associados ao baixo custo e grande disponibilidade (FONSECA, 2001). Vários são os materiais que podem ser usados na sua composição original ou combinados para a produção de mudas de espécies florestais. Esses materiais devem apresentar características físicas e químicas que proporcionem o desenvolvimento adequado das mudas, permitindo boa formação do sistema radicular e da parte aérea da planta.
Segundo Teles et al. (1999), o uso de lodo de esgoto no processo de produção de mudas de espécies florestais é alternativa viável como fonte de matéria orgânica e de nutrientes para as plantas. Trabalhos realizados com lodo de esgoto mostraram resultados satisfatórios como componente orgânico de substratos. Cunha et al. (2006), avaliando o desenvolvimento de mudas de Acacia mangium e Acacia auriculiformis, verificaram que o substrato composto de lodo de esgoto (100\%), com sementes inoculadas com bactérias do gênero Rhizobium, proporcionou maior desenvolvimento às mudas quando comparado com as misturas de solo, areia lavada e esterco bovino (1:1:1, v:v) e solo com areia lavada e lodo de esgoto na mesma proporção. Morais et al. (1997), comparando esterco bovino, lodo de esgoto e acículas de pinus, comprovaram que o melhor crescimento em diâmetro do colo e altura de mudas de cedro (Cedrela fissilis Vell) na fase de viveiro foi obtido em substrato que continha a mistura $70 \%$ de solo sem adubação $+30 \%$ de lodo. Resultados positivos e economia de fertilizantes também foram obtidos em estudos com esse resíduo na produção de mudas de Pinus caribaea (TRIGUEIRO; GUERRINI, 2002) e de Eucalyptus grandis (TRIGUEIRO; GUERRINI, 2003).

Segundo Bonnet et al. (2002), o lodo de esgoto compostado é um excelente componente de substratos, capaz de substituir totalmente o substrato comercial com crescimento compatível das mudas para as espécies testadas (Eucalyptus viminalis, Schinus terebinthifolius e Mimosa scabrella). Esses autores concluíram ainda, em termos de qualidade biométrica e nutricional de mudas, que esse resíduo se mostrou particularmente interessante em percentuais entre $30 \%$ e $60 \%$ do substrato.

O objetivo deste trabalho foi avaliar o desenvolvimento de mudas de aroeira-pimenteira (Schinus terebinthifolius Raddi), produzidas com substratos contendo lodo de esgoto como fonte de matéria orgânica e de nutrientes.

\section{MATERIAL E MÉTODOS}

O experimento foi conduzido no viveiro de produção de mudas do Departamento de Recursos Naturais/Ciências Florestais na Fazenda Experimental Lageado, pertencente à Faculdade de Ciências Agronômicas FCA/UNESP, no Município de Botucatu, 
SP, localizada nas coordenadas geográficas de $22^{\circ} 52^{\prime} 47^{\prime \prime}$ latitude $S, 48^{\circ} 25^{\prime} 12^{\prime \prime}$ longitude W e altitude de $810 \mathrm{~m}$.

As sementes da aroeira-pimenteira (Schinus terebinthifolius Raddi) foram colhidas na Fazenda Experimental, não recebendo qualquer tipo de prétratamento.

Odelineamento experimental utilizado foi o inteiramente casualizado, com seis tratamentos e quatro repetições, sendo cada repetição constituída por uma unidade experimental com 30 plantas. Os tratamentos foram compostos pela mistura de lodo de esgoto (LE) e casca de arroz carbonizada (CAC), nas seguintes proporções: $\mathrm{T} 1=80 \% \mathrm{LE}+20 \% \mathrm{CAC} ; \mathrm{T} 2=70 \% \mathrm{LE}+30 \% \mathrm{CAC}$; $\mathrm{T} 3=60 \% \mathrm{LE}+40 \% \mathrm{CAC}$; $\mathrm{T} 4=50 \% \mathrm{LE}+50 \% \mathrm{CAC}$; e T5 $=40 \% \mathrm{LE}+60 \% \mathrm{CAC}$. A testemunha absoluta foi composta por $100 \%$ de substrato comercial (SC).

O lodo utilizado para compor os substratos foi proveniente da Estação de Tratamento de Esgoto da SABESP, localizada na cidade de Franca, SP. Esse material é produto da digestão anaeróbia do lodo de esgoto residencial e industrial da cidade, sendo utilizado no processo de desidratação um polieletrólito catiônico adicionado à massa do lodo. $\mathrm{O}$ substrato comercial utilizado como testemunha apresenta em sua composição $60 \%$ de composto de casca de pinus, $15 \%$ de vermiculita e $25 \%$ de húmus e terra vegetal.

Após a mistura dos materiais, realizou-se a caracterização química e física dos substratos obtidos, cujos resultados são apresentados nas Tabelas 1 e 2 . Para caracterização das propriedades físicas dos substratos, usou-se a metodologia proposta por Silva (1998).

A semeadura foi realizada em tubetes com capacidade volumétrica de $50 \mathrm{~cm}^{3}$, em que foram colocadas de quatro a cinco sementes por recipiente. Posteriormente, os tubetes foram levados para a câmara de germinação com umidade mantida a $80 \%$ e luminosidade controlada, em que permaneceram até as plântulas atingirem, em média, $3 \mathrm{~cm}$ de altura, quando foi realizado o desbaste, deixando-se apenas uma planta por tubete. Após essa operação de desbaste, os tubetes foram transferidos para a casa de vegetação com sistema de irrigação por aspersão, em que foi aplicada uma lâmina de $7 \mathrm{~mm}$ de água dia $^{-1}$. A fertilização das mudas foi realizada seguindo o sistema usado no viveiro, que consistiu em duas aplicações semanais de nitrato de potássio e nitrato de amônio na dosagem de $2 \mathrm{~g} \mathrm{~L}^{-1}$, com a aplicação de $1 \mathrm{~L}$ de solução por parcela.

Tabela 1 - Atributos químicos dos substratos nos diversos tratamentos.

Table 1 - Chemical attributes of the substrate in the several treatments.

\begin{tabular}{cccccccccccccccccc}
\hline Trat. & $\mathrm{N}$ & $\mathrm{P}_{2} \mathrm{O}_{5}$ & $\mathrm{~K}_{2} \mathrm{O}$ & $\mathrm{MO}$ & $\mathrm{C}$ & $\mathrm{Ca}$ & $\mathrm{Mg}$ & $\mathrm{S}$ & $\mathrm{C} / \mathrm{N}$ & $\mathrm{Na}$ & $\mathrm{Zn}$ & $\mathrm{Cu}$ & $\mathrm{Mn}$ & $\mathrm{Fe}$ & $\mathrm{pH}$ \\
\hline $80 / 20^{1}$ & 5,14 & 2,48 & 0,13 & 61,0 & 33,9 & 1,41 & 0,21 & 1,39 & 7 & 0,113 & 810,98 & 202,13 & 171,60 & 18040 & 6,30 \\
$70 / 30$ & 4,87 & 2,26 & 0,15 & 59,5 & 32,9 & 1,37 & 0,20 & 0,88 & 7 & 0,103 & 789,53 & 190,58 & 181,50 & 16335 & 6,33 \\
$60 / 40$ & 4,92 & 2,09 & 0,23 & 60,0 & 33,4 & 1,30 & 0,16 & 1,09 & 7 & 0,115 & 782,10 & 189,75 & 187,28 & 14671 & 6,38 \\
$50 / 50$ & 4,75 & 1,99 & 0,22 & 59,5 & 33,1 & 1,11 & 0,18 & 1,37 & 8 & 0,115 & 660,00 & 153,45 & 197,18 & 12348 & 6,38 \\
$40 / 60$ & 4,51 & 1,76 & 0,23 & 58,8 & 32,7 & 1,23 & 0,16 & 0,67 & 8 & 0,110 & 720,23 & 169,95 & 219,45 & 12664 & 6,28 \\
$\mathrm{SC}$ & 0,63 & 0,41 & 0,15 & 28,5 & 18,8 & 0,82 & 0,15 & 0,15 & 28 & 0,095 & 154,28 & 33,00 & 169,95 & 3039 & 5,03 \\
\hline
\end{tabular}

${ }^{1} \mathrm{LE} / \mathrm{CAC}=$ lodo de esgoto/casca de arroz carbonizada.

${ }^{1} \mathrm{LE} / \mathrm{CAC}=$ sewage sludge/carbonized rice hull.

Tabela 2 - Atributos físicos dos substratos nos diversos tratamentos.

Table 2 - Physical attributes of the substrate in the several treatments.

\begin{tabular}{|c|c|c|c|c|c|c|}
\hline Trat. & Macro poros & Microporos & Porosidade Total & $\begin{array}{l}\text { Capacidade de } \\
\text { retenção de água }\end{array}$ & $\begin{array}{l}\text { Densidade } \\
\text { aparente }\end{array}$ & $\begin{array}{l}\mathrm{g} \text { de água por } \\
\mathrm{g} \text { de substrato }\end{array}$ \\
\hline & & $(\%)$ & & $\mathrm{mL} 50 \mathrm{~cm}^{-3}$ & $\mathrm{~g} \mathrm{~cm}^{-3}$ & \\
\hline $80 / 20^{1}$ & 16,09 & 48,69 & 64,79 & 24,25 & 0,53 & 0,93 \\
\hline $70 / 30$ & 21,77 & 44,36 & 67,18 & 22,09 & 0,48 & 0,92 \\
\hline $60 / 40$ & 22,81 & 45,05 & 66,82 & 22,43 & 0,47 & 0,97 \\
\hline $50 / 50$ & 22,85 & 46,79 & 69,64 & 23,30 & 0,44 & 1,07 \\
\hline $40 / 60$ & 32,00 & 41,78 & 73,78 & 20,80 & 0,40 & 1,06 \\
\hline $\mathrm{SC}$ & 20,42 & 53,61 & 74,03 & 26,67 & 0,28 & 1,89 \\
\hline
\end{tabular}

${ }^{1} \mathrm{LE} / \mathrm{CAC}=$ lodo de esgoto/casca de arroz carbonizada.

${ }^{1}$ LE/CAC = sewage sludge/carbonized rice hull. 
As medidas de altura das mudas foram realizadas em quatro épocas, a partir dos 60 até os 180 dias após a semeadura. O diâmetro do colo foi medido com paquímetro digital, nas mesmas épocas, exceto 60 dias após a semeadura. Depois da última avaliação de altura e diâmetro (180 dias após a semeadura), as mudas foram retiradas dos tubetes, com remoção do substrato e lavagem cuidadosa do sistema radicular. As plantas foram cortadas à altura do colo, para separar parte aérea e raiz. As raízes e partes aéreas foram secas em estufa, com circulação forçada de ar a $60^{\circ} \mathrm{C}$, para determinação da matéria seca, em que a parte aérea e o sistema radicular foram triturados para análise química, utilizando-se a metodologia descrita por Malavolta et al. (1997). O acúmulo de nutrientes pelas mudas foi obtido através da multiplicação da concentração de nutrientes pela quantidade de matéria seca da parte aérea e do sistema radicular. Foi observado, também, o índice de sobrevivência dado, em termos percentuais, pela relação entre o número de plantas vivas no início e final do experimento.

Os resultados foram submetidos à análise de variância, avaliando-se o efeito dos tratamentos dentro de cada época de avaliação, e as médias, comparadas por meio do teste de Tukey a $5 \%$ de probabilidade, com o uso do Programa SISVAR.

\section{RESULTADOS}

De acordo com os resultados da análise físico-química dos substratos, observou-se que o LE continha altos teores de nutrientes, com destaque para o nitrogênio e fósforo, enquanto a CAC possuía teor de potássio mais elevado. Ambos os componentes possuíam alto percentual de carbono, e os substratos com maiores quantidades de $\mathrm{CAC}$ apresentaram relação $\mathrm{C} / \mathrm{N}$ mais alta, devido ao baixo teor de nitrogênio presente nesse componente. Na análise de micronutrientes, o LE apresentou teores mais elevados de $\mathrm{Zn}, \mathrm{Cu}$ e Fe, enquanto a CAC, teor mais elevado apenas de Mn. Todos os tratamentos proporcionaram valores de $\mathrm{pH}$ dentro da faixa considerada adequada para o desenvolvimento de mudas, que é de 5,5 a 6,5, segundo Valeri e Corradini (2000). Com relação à caracterização física dos substratos, os resultados revelaram que, à medida que se elevou a dose de LE na mistura, ocorreu aumento da sua densidade e, consequentemente, redução da macroporosidade e aumento da microporosidade do substrato, o que proporcionou maior capacidade de retenção de água.

Considerando as características químicas apresentadas pelos materiais, ocorreu, inicialmente, maior crescimento em altura das plantas submetidas aos tratamentos com LE. Porém, esse comportamento foi alterado nas medições subsequentes, possivelmente devido à introdução da fertirrigação (Tabela 3). Ao

Tabela 3 - Parâmetros de crescimento: altura (H) e diâmetro do colo (D) em diferentes épocas de avaliação; matéria seca da parte aérea; matéria seca de raiz; e sobrevivência de mudas de aroeira-pimenteira crescidas sob diferentes misturas de lodo de esgoto e casca de arroz no substrato.

Table 3 - Growth parameters: high (H) and collar diameter (D) at different times of assessment; dry matter of shoots and roots and survival of Brazilian pepper seedlings grown under different proportions of sewage sludge and carbonized rice hull in the substrate.

\begin{tabular}{|c|c|c|c|c|c|c|c|c|c|c|c|c|}
\hline \multirow[t]{3}{*}{ Trat. } & $\mathrm{H}$ & $\mathrm{H}$ & $\mathrm{D}$ & $\mathrm{H}$ & $\mathrm{D}$ & $\mathrm{H}$ & $\mathrm{D}$ & \multirow[t]{3}{*}{$\mathrm{H} / \mathrm{D}$} & \multirow{3}{*}{$\begin{array}{c}\text { Matéria } \\
\text { seca da } \\
\text { parte aérea }\end{array}$} & \multirow{3}{*}{$\begin{array}{c}\text { Matéria } \\
\text { seca de } \\
\text { raiz } \\
\end{array}$} & \multicolumn{2}{|c|}{ Sobrevivência } \\
\hline & \multicolumn{7}{|c|}{$(\mathrm{cm})$} & & & & & \\
\hline & \multicolumn{7}{|c|}{ Dias após a semeadura } & & & & Germinação & Expedição \\
\hline- & 60 & \multicolumn{2}{|c|}{90} & \multicolumn{2}{|c|}{120} & \multicolumn{3}{|c|}{180} & \multicolumn{2}{|c|}{ g planta $^{-1}$} & \multicolumn{2}{|c|}{$\%$} \\
\hline $80 / 20^{1}$ & $3,4 \mathrm{c}$ & $5,6 \mathrm{c}$ & $1,4 \mathrm{~b}$ & $11,5 \mathrm{~d}$ & $2,1 \mathrm{~d}$ & $25,9 \mathrm{c}$ & $3,3 \mathrm{c}$ & 7,8 & $2,03 b c$ & $0,60 \mathrm{c}$ & 65,8 & 64,2 \\
\hline $70 / 30$ & $4,0 \mathrm{ab}$ & $5,9 \mathrm{bc}$ & $1,4 \mathrm{~b}$ & $13,9 \mathrm{~cd}$ & $2,3 \mathrm{~cd}$ & $26,9 \mathrm{c}$ & $3,3 \mathrm{c}$ & 8,1 & $1,93 \mathrm{c}$ & $0,60 \mathrm{c}$ & 99,2 & 90,9 \\
\hline $60 / 40$ & $4,2 \mathrm{ab}$ & $7,7 \mathrm{ab}$ & $1,6 \mathrm{~b}$ & $17,5 \mathrm{~b}$ & $2,6 b$ & $31,4 \mathrm{~b}$ & $3,6 b c$ & 8,7 & $2,35 b$ & $0,78 b$ & 98,4 & 91,7 \\
\hline $50 / 50$ & $4,1 \mathrm{ab}$ & $6,5 b c$ & $1,5 \mathrm{~b}$ & $15,3 \mathrm{bc}$ & $2,4 \mathrm{bc}$ & $30,8 b$ & $3,5 b c$ & 8,8 & $2,28 b c$ & $0,73 \mathrm{bc}$ & 94,2 & 91,7 \\
\hline $40 / 60$ & $4,4 \mathrm{a}$ & $7,5 \mathrm{bc}$ & $1,6 \mathrm{~b}$ & $17,1 \mathrm{bc}$ & $2,6 \mathrm{bc}$ & $31,8 \mathrm{~b}$ & $3,7 b$ & 8,5 & $2,20 \mathrm{bc}$ & $0,80 \mathrm{~b}$ & 100,0 & 96,7 \\
\hline $\mathrm{SC}$ & $3,8 \mathrm{bc}$ & $9,5 \mathrm{a}$ & $2,0 \mathrm{a}$ & $22,9 \mathrm{a}$ & $3,4 \mathrm{a}$ & $43,6 a$ & $4,8^{\mathrm{a}}$ & 9,1 & $3,78 \mathrm{a}$ & $1,08 \mathrm{a}$ & 98,4 & 96,7 \\
\hline $\mathrm{F}$ & $* *$ & $* *$ & $* *$ & $* *$ & ** & ** & ** & $\mathrm{ns}$ & $* *$ & $* *$ & * & $\mathrm{ns}$ \\
\hline $\mathrm{CV} \%$ & 5,6 & 11,8 & 8,7 & 9,1 & 5,2 & 6,4 & 4,3 & 8,5 & 7,4 & 9,9 & 16,9 & 18,00 \\
\hline
\end{tabular}

${ }^{1} \mathrm{LE} / \mathrm{CAC}=$ lodo de esgoto/casca de arroz carbonizada; $* *$ Significativo a $1 \%$ de probabilidade; ns - Não significativo; Médias seguidas de mesma letra na coluna não diferem entre si, pelo teste de Tukey $(\mathrm{P}>0,05)$; e $\mathrm{SC}=$ substrato comercial.

${ }^{1}$ LE / CAC = sewage sludge/carbonized rice hull; ** significant at $1 \%$ of probability; ns - not significant; Means followed by same letter in the column do not differ by Tukey test $(P>0.05)$; and $S C=$ commercial substrate.

Revista Árvore, Viçosa-MG, v.38, n.4, p.657-665, 2014 
final do experimento, 180 dias após a semeadura, observou-se que nenhum dos tratamentos com LE superou a testemunha em altura ou em diâmetro do colo. Os tratamentos com as relações de 60/40, 50/ 50 e 40/60 de LE/CAC proporcionaram melhores resultados, tanto para altura de planta quanto para diâmetro, não diferindo estatisticamente entre si.

Para a matéria seca da parte aérea e do sistema radicular, também foram observados resultados superiores na testemunha (Tabela 3). Na relação entre peso de matéria seca do sistema radicular e da parte aérea, não houve diferenças significativas entre os diferentes substratos. De acordo com o índice de sobrevivência de mudas, o tratamento contendo uma proporção de $80 \%$ de LE e $20 \%$ da CAC foi prejudicial à germinação e sobrevivência das mudas por ocasião da expedição (Tabela 3 ).

As concentrações de nutrientes na parte aérea e na raiz, apresentadas na Tabela 4 , foram estreitamente relacionadas com os teores de nutrientes presentes nos substratos. O aumento dos teores de nutrientes nos substratos corresponde ao da quantidade de LE utilizada, exceto para os de K e Mn, que estão presentes em quantidades muito pequenas nesse resíduo. $\mathrm{O}$ fornecimento de nutrientes às plantas do tratamentotestemunha ocorreu, principalmente, via fertirrigação, pois o substrato comercial usado apresentou teores de nutrientes muito inferiores aos substratos com LE.

As concentrações de $\mathrm{N}, \mathrm{P}, \mathrm{K}, \mathrm{Ca}, \mathrm{S}, \mathrm{Cu}, \mathrm{Fe}, \mathrm{Mn}$ e Zn na parte aérea das plantas dos tratamentos com LE foram estatisticamente superiores às concentrações desses nutrientes nas plantas da testemunha (Tabela 4). A concentração de nutrientes nas raízes apresentou o mesmo comportamento, com exceção do $\mathrm{Ke} \mathrm{Ca}$, que não diferiram estatisticamente da testemunha. As concentrações de B nas raízes sofreram pouca influência do substrato utilizado.

O maior acúmulo de matéria seca das plantas da testemunha não refletiu no maior acúmulo de todos os nutrientes pelas mudas, pois esse aumento foi

Tabela 4 - Concentração de nutrientes na parte aérea e no sistema radicular de mudas de aroeira-pimenteira crescidas sob diversas misturas de lodo de esgoto e casca de arroz no substrato.

Table 4 - Nutrients concentration in shoots and roots of Brazilian pepper seedlings grown under different proportions of sewage sludge and carbonized rice hull in the substrate.

\begin{tabular}{|c|c|c|c|c|c|c|c|c|c|c|c|}
\hline \multirow[t]{2}{*}{ Trat. } & \multirow[b]{2}{*}{$\mathrm{N}$} & \multicolumn{5}{|c|}{ Macronutrientes } & \multicolumn{5}{|c|}{ Micronutrientes } \\
\hline & & $\mathrm{P}$ & $\mathrm{K}$ & $\mathrm{Ca}$ & $\mathrm{Mg}$ & $\mathrm{S}$ & $\mathrm{B}$ & $\mathrm{Cu}$ & $\mathrm{Fe}$ & $\mathrm{Mn}$ & $\mathrm{Zn}$ \\
\hline & & \multicolumn{5}{|c|}{$\mathrm{g} \mathrm{kg}^{-1}$} & \multicolumn{5}{|c|}{$\mathrm{mg} \mathrm{kg}^{-1}$} \\
\hline \multicolumn{12}{|c|}{ Parte aérea } \\
\hline $80 / 20^{1}$ & $22 \mathrm{ab}$ & $3,2 \mathrm{a}$ & $18 \mathrm{ab}$ & $9,4 \mathrm{a}$ & $1,6 \mathrm{a}$ & $2,8^{\mathrm{a}}$ & $18 \mathrm{ab}$ & $5 \mathrm{a}$ & $85 a$ & $99 \mathrm{ab}$ & $66 b$ \\
\hline $70 / 30$ & $24 \mathrm{a}$ & $3,1 \mathrm{a}$ & $20 a$ & $9,3 \mathrm{a}$ & $1,5 \mathrm{ab}$ & $2,4 \mathrm{ab}$ & $17 \mathrm{ab}$ & $5 \mathrm{a}$ & $78 \mathrm{ab}$ & $108 \mathrm{a}$ & $77 \mathrm{ab}$ \\
\hline $60 / 40$ & $22 \mathrm{ab}$ & $2,6 a b$ & $17 \mathrm{ab}$ & $9,0 \mathrm{ab}$ & $1,5 \mathrm{ab}$ & $1,8 \mathrm{bc}$ & $16 a b$ & $5 \mathrm{a}$ & $71 \mathrm{ab}$ & $95 \mathrm{ab}$ & $72 \mathrm{ab}$ \\
\hline $50 / 50$ & $23 a$ & $2,7 \mathrm{ab}$ & $18 \mathrm{ab}$ & $8,8 \mathrm{ab}$ & $1,4 \mathrm{ab}$ & $2,0 \mathrm{bc}$ & $16 \mathrm{ab}$ & $5 \mathrm{a}$ & $70 \mathrm{~b}$ & $103 \mathrm{ab}$ & $84 a$ \\
\hline $40 / 60$ & $23 a$ & $2,2 b$ & $18 \mathrm{ab}$ & $7,9 \mathrm{bc}$ & $1,3 b$ & $1,6 \mathrm{~cd}$ & $16 b$ & $4 a$ & $69 b$ & $88 b$ & $75 \mathrm{ab}$ \\
\hline SC18b & $1,5 \mathrm{c}$ & $15 b$ & $6,9 \mathrm{c}$ & $1,5 \mathrm{ab}$ & $1,0 \mathrm{~d}$ & $19 \mathrm{a}$ & $2 b$ & $49 \mathrm{c}$ & $31 \mathrm{c}$ & $17 \mathrm{c}$ & \\
\hline $\mathrm{F} \quad *$ & $* *$ & $*$ & $* *$ & $*$ & $* *$ & * & $* *$ & $* *$ & $* *$ & $* *$ & \\
\hline CV\% & 8,5 & 9,8 & 9,7 & 5,7 & 7,5 & 14,2 & 7,2 & 13,3 & 9,2 & 8,9 & 11,9 \\
\hline \multicolumn{12}{|c|}{ Sistema radicular } \\
\hline $80 / 20^{1}$ & $23 a b$ & $3,6 a$ & $15 \mathrm{a}$ & $7,9 \mathrm{a}$ & $1,6 a b$ & $3,1^{\mathrm{a}}$ & 25 & $57 \mathrm{ab}$ & $2735 a$ & $78 \mathrm{a}$ & $640 a$ \\
\hline $70 / 30$ & $23 a$ & $3,7 \mathrm{a}$ & $15 \mathrm{a}$ & $7,7 \mathrm{ab}$ & $1,3 \mathrm{abc}$ & $2,9 \mathrm{ab}$ & 21 & $57 \mathrm{a}$ & $2820 a$ & $83 a$ & $700 a$ \\
\hline $60 / 40$ & $21 \mathrm{ab}$ & $3,3 \mathrm{a}$ & $13 a$ & $7,0 \mathrm{ab}$ & $1,3 \mathrm{abc}$ & $2,4 \mathrm{abc}$ & 23 & $49 \mathrm{ab}$ & $2905 a$ & $74 \mathrm{ab}$ & $640 a$ \\
\hline $50 / 50$ & $21 \mathrm{ab}$ & $3,3 \mathrm{a}$ & $13 a$ & $6,8 \mathrm{ab}$ & $1,2 \mathrm{bc}$ & $2,1 \mathrm{bc}$ & 22 & $50 \mathrm{ab}$ & $2465 \mathrm{ab}$ & $80 a$ & $715 a$ \\
\hline $40 / 60$ & $19 b$ & $2,8 \mathrm{~b}$ & $13 a$ & $5,4 \mathrm{~b}$ & $1,0 \mathrm{c}$ & $1,7 \mathrm{~cd}$ & 21 & $34 b$ & $1705 \mathrm{ab}$ & $64 a b$ & $480 \mathrm{a}$ \\
\hline $\mathrm{SC} 14 \mathrm{c}$ & $1,7 \mathrm{c}$ & $14 \mathrm{a}$ & $6,6 \mathrm{ab}$ & $1,7 \mathrm{a}$ & $1,2 \mathrm{~d}$ & 18 & $10 \mathrm{c}$ & $751 b$ & $35 b$ & $493 b$ & \\
\hline $\mathrm{F} * *$ & $* *$ & $*$ & $*$ & $*$ & $* *$ & $\mathrm{~ns}$ & $* *$ & $*$ & $*$ & $* *$ & \\
\hline CV\% & 9,9 & 7,2 & 8,5 & 14,8 & 14,2 & 15,4 & 14,1 & 24,3 & 36,1 & 27,2 & 22,0 \\
\hline
\end{tabular}

${ }^{1} \mathrm{LE} / \mathrm{CAC}=1$ lodo de esgoto/casca de arroz carbonizada; $* *$ significativo ao nível de $1 \%$ de probabilidade; $*$ significativo ao nível de $5 \%$ de probabilidade; ns - Não significativo; Médias seguidas de mesma letra na coluna não diferem estatisticamente entre si, pelo teste de Tukey $(\mathrm{P}>0,05)$; e $\mathrm{SC}=$ substrato comercial

${ }^{1} \mathrm{LE} / \mathrm{CAC}=$ sewage sludge/carbonized rice hull; ** significant at $1 \%$ of probability; * significant at $5 \%$ of probability; ns - not significant; Means followed by the same letter in the column are not statistically different by Tukey test (P>0.05); and SC = commercial substrate. 
observado apenas para N, K, Ca, Mg e B na parte aérea e $\mathrm{K}$ e Mg nas raízes (Tabela 5). De forma geral, não se obtiveram diferenças significativas no acúmulo de nutrientes, tanto na parte aérea quanto nas raízes, entre os tratamentos com LE (Tabela 5).

\section{DISCUSSÃO}

Um estudo realizado em um viveiro de mudas limitase a reconhecer a qualidade destas por meio da análise de alguns parâmetros. De acordo com Gomes et al. (2002), o diâmetro do colo das mudas é considerado um dos mais importantes parâmetros para estimar sobrevivência de mudas no campo de diferentes espécies florestais. Assim, essa é a variável mais indicada para auxiliar na definição das doses de fertilizantes a serem aplicadas, objetivando a produção de mudas arbóreas de boa qualidade (CARNEIRO, 1995), inclusive da aroeira (JOSÉ et al., 2005). Segundo Caldeiras et al. (2008), a altura e diâmetro do colo das mudas plantadas no campo devem ter de 30 a $35 \mathrm{~cm}$ e de 2,2 a $2,5 \mathrm{~cm}$, respectivamente. Nesse experimento, as mudas produzidas nos diferentes substratos apresentaram qualidade desejável dessas características morfológicas, principalmente quando observado o diâmetro do colo, cujo menor valor foi de 3,3 cm (Tabela 3 ).

Quantidades maiores de lodo de esgoto proporcionaram menor crescimento em altura e diâmetro do colo (Tabela 3). Esses resultados podem estar associados à menor quantidade de macroporos nesses tratamentos, que têm como consequência menor aeração. Estudos realizados por Caldeira et al. (2008), utilizando também a aroeira-pimenteira, demonstraram que mudas produzidas com $100 \%$ de composto orgânico não tiveram bom desenvolvimento. Nóbrega et al. (2007), avaliando diferentes proporções de lodo de esgoto em aroeira, verificaram que o máximo diâmetro de colo foi obtido quando adicionados $37 \%$ do resíduo. Para altura de plantas, Nóbrega et al. (2007) obtiveram a mesma tendência observada neste trabalho, ou seja, de redução da altura com doses maiores de lodo.

Tabela 5 - Acúmulo de nutrientes na parte aérea e no sistema radicular de mudas de aroeira- pimenteira crescidas sob diversas misturas de lodo de esgoto e casca de arroz no substrato.

Table 5 - Nutrient accumulation in shoots and roots of Brazilian pepper seedlings grown under different proportions of sewage sludge and carbonized rice hull in the substrate.

\begin{tabular}{|c|c|c|c|c|c|c|c|c|c|c|c|}
\hline \multirow[t]{2}{*}{ Trat. } & & \multicolumn{5}{|c|}{ Macronutrientes } & \multicolumn{5}{|c|}{ Micronutrientes } \\
\hline & $\mathrm{N}$ & $\mathrm{P}$ & $\mathrm{K}$ & $\mathrm{Ca}$ & $\mathrm{Mg}$ & $\mathrm{S}$ & $\mathrm{B}$ & $\mathrm{Cu}$ & $\mathrm{Fe}$ & Mn & $\mathrm{Zn}$ \\
\hline & & \multicolumn{5}{|c|}{ mg planta ${ }^{-1}$} & \multicolumn{5}{|c|}{$\mu \mathrm{g}$ planta $^{-1}$} \\
\hline \multicolumn{12}{|c|}{ Parte aérea } \\
\hline $80 / 201$ & $44 b$ & $6,3 \mathrm{a}$ & $36 b$ & $19 \mathrm{bc}$ & $3,2 b$ & $5,6^{\mathrm{a}}$ & $37 b$ & $10 \mathrm{ab}$ & $171 \mathrm{ab}$ & $200 \mathrm{ab}$ & $132 b$ \\
\hline $70 / 30$ & $45 b$ & $6,0 \mathrm{ab}$ & $38 b$ & $18 \mathrm{bc}$ & $2,9 b$ & $4,6 a b$ & $32 b$ & $9 b$ & $149 b$ & $207 \mathrm{ab}$ & $148 \mathrm{ab}$ \\
\hline $60 / 40$ & $52 b$ & $6,2 \mathrm{a}$ & $40 b$ & $21 b$ & $3,4 b$ & $4,3 a b$ & $39 b$ & $12 \mathrm{a}$ & $167 \mathrm{ab}$ & $224 \mathrm{ab}$ & $169 \mathrm{ab}$ \\
\hline $50 / 50$ & $53 b$ & $6,3 \mathrm{a}$ & $42 b$ & $20 b c$ & $3,2 b$ & $4,5 \mathrm{ab}$ & $38 b$ & $11 \mathrm{ab}$ & $158 \mathrm{ab}$ & $235 \mathrm{a}$ & $192 \mathrm{a}$ \\
\hline $40 / 60$ & $49 b$ & $4,8 b$ & $39 b$ & $17 \mathrm{c}$ & $2,9 b$ & $3,5 b$ & $35 b$ & $9 \mathrm{ab}$ & $151 \mathrm{~b}$ & $190 b$ & $163 \mathrm{ab}$ \\
\hline SC69a & $5,4 \mathrm{ab}$ & $58 \mathrm{a}$ & $26^{\mathrm{a}}$ & $5,8 \mathrm{a}$ & $3,8 \mathrm{~b}$ & $72 \mathrm{a}$ & $6 \mathrm{c}$ & $187 \mathrm{a}$ & $118 \mathrm{c}$ & $62 \mathrm{c}$ & \\
\hline $\mathrm{F} * *$ & $*$ & $* *$ & $* *$ & $* *$ & $*$ & $* *$ & $* *$ & $*$ & $* *$ & $* *$ & \\
\hline $\mathrm{CV} \%$ & 10,7 & 9,4 & 11,8 & 8,2 & 9,9 & 14,8 & 9,1 & 13,6 & 8,3 & 9,5 & 13,9 \\
\hline \multicolumn{12}{|c|}{ Sistema radicular } \\
\hline $80 / 201$ & 15 & $2,3 \mathrm{ab}$ & $10 \mathrm{~b}$ & $5,1 \mathrm{ab}$ & $1,0 \mathrm{~b}$ & $2,0 \mathrm{a}$ & $16 \mathrm{ab}$ & $36 a$ & $1750 \mathrm{ab}$ & 50 & $409 a$ \\
\hline $70 / 30$ & 14 & $2,2 \mathrm{ab}$ & $9 b$ & $4,5 \mathrm{ab}$ & $0,8 \mathrm{~b}$ & $1,7 \mathrm{ab}$ & $12 b$ & $33 a$ & $1615 \mathrm{ab}$ & 48 & $408 \mathrm{a}$ \\
\hline $60 / 40$ & 16 & $2,6 \mathrm{a}$ & $10 b$ & $5,4 \mathrm{ab}$ & $1,0 \mathrm{~b}$ & $1,8 \mathrm{ab}$ & $17 \mathrm{ab}$ & $37 \mathrm{a}$ & $2211 \mathrm{a}$ & 57 & $490 \mathrm{a}$ \\
\hline $50 / 50$ & 15 & $2,4 \mathrm{a}$ & $9 b$ & $4,9 \mathrm{ab}$ & $0,9 b$ & $1,5 \mathrm{ab}$ & $16 \mathrm{ab}$ & $36 a$ & $1788 \mathrm{ab}$ & 58 & $516 a$ \\
\hline $40 / 60$ & 15 & $2,2 \mathrm{ab}$ & $10 b$ & $4,3 b$ & $0,8 \mathrm{~b}$ & $1,4 \mathrm{ab}$ & $16 \mathrm{ab}$ & $27 \mathrm{ab}$ & $1366 \mathrm{ab}$ & 51 & $383 a$ \\
\hline $\mathrm{SC} 14$ & $1,8 \mathrm{~b}$ & $15 \mathrm{a}$ & $7,0 \mathrm{a}$ & $1,8 \mathrm{a}$ & $1,3 b$ & $19 a$ & $10 \mathrm{~b}$ & $810 \mathrm{~b}$ & 37 & $53 b$ & \\
\hline $\mathrm{F} \mathrm{ns}$ & $*$ & $* *$ & * & $* *$ & $*$ & $*$ & * & $*$ & $\mathrm{~ns}$ & $* *$ & \\
\hline $\mathrm{CV} \%$ & 13,2 & 9,9 & 9,6 & 17,0 & 17,9 & 15,5 & 15,6 & 26,5 & 35,3 & 30,3 & 26,1 \\
\hline
\end{tabular}

${ }^{1} \mathrm{LE} / \mathrm{CAC}=$ lodo de esgoto/casca de arroz carbonizada; $* *$ Significativo a $1 \%$ de probabilidade; $*$ Significativo a $5 \%$ de probabilidade; ns Não significativo; Médias seguidas de mesma letra na coluna não diferem estatisticamente entre si, pelo teste de Tukey (P $>0,05)$; e $\mathrm{SC}$ - Substrato comercial.

${ }^{1}$ LE / CAC = sewage sludge/carbonized rice hull; ** significant at $1 \%$ of probability; * significant at $5 \%$ of probability; ns - not significant; Means followed by the same letter in the column are not statistically different by Tukey test (P>0.05); and SC = commercial substrate.

Revista Árvore, Viçosa-MG, v.38, n.4, p.657-665, 2014 
Muitas vezes, utiliza-se a combinação da altura e diâmetro, gerando um índice que fornece informações sobre quanto a muda está delgada. De acordo com Carneiro (1995), a relação altura/diâmetro deve situarse entre 5,4 e 8,1. Apesar de a análise desse parâmetro não ter mostrado diferença significativa entre tratamentos e a testemunha (Tabela 3), observou-se tendência a valores acima do limite considerado como adequando, revelando que o experimento poderia ter sido finalizado antes dos 180 dias. Segundo Nóbrega et al. (2007), as mudas que apresentam altura excessiva, não acompanhada de aumento do diâmetro do colo, ficam sensíveis aos fatores climáticos, como vento e chuva.

Os tratamentos com 70 e $80 \%$ de LE proporcionaram menor desenvolvimento do sistema radicular (Tabela 3), possivelmente pela maior densidade apresentada por esses substratos, uma vez que substratos com alta densidade diminuem a drenagem e, consequentemente, a aeração, criando um ambiente pouco propício ao desenvolvimento do sistema radicular. Corroborando esses resultados, Silva et al. (2012) observaram que os substratos com maior porosidade total promovem maior qualidade do sistema radicular e, por consequência, gera mudas com maior massa seca de parte aérea e radicular. Já Mauad et al. (2004) relataram que, por apresentar menor densidade proveniente da maior porcentagem de macroporos, a casca de arroz carbonizada proporciona melhor escoamento de excesso de água e favorece o desenvolvimento do sistema radicular.

O aumento da densidade, provocado por maiores quantidades de LE, está, portanto, diretamente relacionado com a redução da porosidade, afetando negativamente, dessa forma, as boas características físicas do substrato, a exemplo da adequada proporção de ar e umidade após a drenagem natural. Trigueiro e Guerrini (2003) verificaram resultados semelhantes quando utilizaram os mesmos substratos para a produção de mudas de eucalipto. Franczak et al. (2008), ao adicionarem doses de lodo de curtume em substrato comercial, também verificaram que altas concentrações desse material orgânico causaram efeito fitotóxico nas plantas de Jacaranda cuspidifolia Mart., reduzindo as taxas de sobrevivência, o que foi atribuído ao endurecimento do substrato, que causou, aparentemente, perda de porosidade.
Sobre os aspectos nutricionais das plantas de aroeira-pimenteira, pode-se observar que aumentos nas concentrações de $\mathrm{P}$ na parte aérea e na raiz são acompanhados de incrementos nas concentrações de $\mathrm{Mg}$, provavelmente devido ao efeito sinérgico entre esses dois nutrientes, conforme relataram também Assis (1995) e Trigueiro e Guerrini (2003).

Não foram observados sintomas visuais de deficiência, ou de toxidez nas plantas de aroeira em nenhuma fase de crescimento, mesmo estando os teores de $\mathrm{Zn}$ e $\mathrm{Cu}$ dos substratos com LE dentro da faixa considerada fitotóxica por Kabata-Pendias e Pendias (1985), ou seja, entre $60-125 \mathrm{mg} \mathrm{kg}^{-1}$ para $\mathrm{Cu}$ e 70 - $400 \mathrm{mg} \mathrm{kg}^{-1}$ para $\mathrm{Zn}$.

Os resultados deste estudo corroboram os encontrados por Trigueiro e Guerrini (2002, 2003), que observam que a produção de mudas usando como substrato o lodo de esgoto em mistura com casca de arroz carbonizada é viável em espécies florestais de rápido crescimento. Em geral, nesses trabalhos altas doses de lodo de esgoto (70 e $80 \%$ ) prejudicaram a formação das mudas. As proporções de lodo de esgoto no substrato devem ser de 40 a $60 \%$, em mistura com casca de arroz carbonizada. Resultados semelhantes foram obtidos por Bonnet (2002), que recomendou o uso de 30 a $60 \%$ de lodo compostado em mistura com substrato comercial para produção de mudas de aroeirapimenteira.

A qualidade de mudas produzidas com substratos contendo lodo de esgoto em sua composição poderá ser comprovada, avaliando-se seu desempenho no campo. Assim, este trabalho deve ser complementado por meio da instalação de experimentos que possam analisar, em longo prazo, o comportamento dessas plantas diante das dificuldades oferecidas pelas fases posteriores à expedição das mudas.

\section{CONCLUSÕES}

Diante dos resultados, pode-se concluir que:

1 - As doses de lodo de esgoto para a produção de mudas de Schinus terebinthifolius (Raddi) em combinação com casca de arroz carbonizada devem situar-se entre 40 e $60 \%$.

2- O uso de lodo de esgoto em viveiros florestais, na composição de substratos, é alternativa viável para a disposição final desse resíduo.

Revista Árvore, Viçosa-MG, v.38, n.4, p.657-665, 2014 


\section{REFERÊNCIAS}

ASSIS, R. P. Nutrição mineral e crescimento de mudas de dendezeiro (Elaeis guineensis Jacq.) em função de diferentes relações entre $\mathrm{K}$, Ca e Mg na solução nutritiva. 1995. $41 \mathrm{f}$. Dissertação (Mestrado em Solos e Nutrição de Plantas) Universidade Federal de Lavras, Lavras, 1995.

BONNET, B. R. P. et al. Effects of substracts composed of biosolids on the production of Eucalyptus viminalis, Schinus terebinthifolius and Mimosa scabrella seedlings and on the nutritional status of Schinus terebinthifolius seedlings. Water Science and

Technology, v.46, n.10, p.239-246, 2002.

CALDEIRA, M. V. W. et al. Uso do resíduo de algodão no substrato para produção de mudas florestais. Revista Acadêmica de Ciências Agrárias e Ambientais, v.6, n.2, p.191-202, 2008.

CARneiro, J. G. A. Produção e controle de qualidade de mudas florestais. Viçosa, MG: Editora Folha de Viçosa, 1995. 451p

CROCE, C. G. G.; CARMO, M. S.; CÂMARA, F. L. A. Desenvolvimento inicial e poder calorífico de duas espécies arbóreas nativas e pioneiras sob tratamentos convencional, orgânico e biodinâmico. Energia na Agricultura, v.22, n.4, p.10-24, 2007.

CUNHA, A. M. et al. Efeito de diferentes substratos sobre o desenvolvimento de mudas de Acacia sp. Revista Árvore, v.30, n.2, p.207$214,2006$.

DURIGAN, G. et al. Sementes e mudas de árvores tropicais. São Paul: Páginas e Letras 1997.65p

GOMES, J. M. et al. Parâmetros morfológicos na avaliação da qualidade de mudas de Eucaliptus grandis. Revista Árvore, v.26, n.6, p.655-664, 2002.

FOnSECA, T. G. Produção de mudas de hortaliças em substratos de diferentes composições com adição de CO2 na água de irrigação. 2001. 72f. Dissertação (Mestrado em Agronomia) - Escola Superior de Agricultura "Luiz de Queiroz", Piracicaba, 2001.
FRANCZAK, D. D. et al. Adição de dosagens de lodo de curtume em substrato comercial para produção de mudas de caroba ( jacaranda cuspidifolia mart.). In: ENCONTRO NACIONAL SOBRE SUBSTRATOS PARA PLANTAS MATERIAIS REGIONAIS COMO SUBSTRATO, 6 , 2008, Fortaleza Anais... Fortaleza: Embrapa Agroindustria Tropical, SEBRAE/CE e UFC, 2008.

JOSÉ, A. C.; DAVIDE, A. C.; OLIVEIRA, S. L. Produção de mudas de aroeira (Schinus terebynthifolius Raddi) para recuperação de áreas degradadas pela mineração de bauxita. Cerne, v.11, n.2, p.187-196, 2005.

KABATA-PENDIAS, A.; PENDIAS, H. Trace elements in soils and plants. Boca Raton: CRc Press, 1985. 315p.

LELES, P. S. S. et al. Qualidade de mudas de quatro espécies florestais produzidas em diferentes tubetes. Floresta e Ambiente, v.13, n.1, p.69-78, 2006.

MALAVOLTA, E.; VITTI, G. C.; OLIVEIRA, S. A. Avaliação do estado nutricional das plantas 2.ed. Piracicaba: Associação Brasileira para Pesquisa da Potassa e do Fosfato, 1997. 319p

MAUAD, M. et al. Enraizamento de estacas de azaléia tratadas com concentrações de ANA em diferentes substratos. Ciência e

Agrotecnologia, v.28, n.4, p.771-777, 2004.

MORAIS, S. M. J. et al. Uso do lodo de esgoto da Corsan - Santa Maria (RS), comparado com outros substratos orgânicos. Sanare, v.6, n.6, p.44-49, 1997.

NÓBREGA, R. S. A. et al. Utilização de biossólido no crescimento inicial de mudas de aroeira (Schinus terebynthifolius Raddi). Revista Árvore, v.31, n.2, p.239-246, 2007.

SILVA, M. R. Caracterização morfológica, fisiológica e nutricional de mudas de Eucalyptus grandis Hill ex Maiden submetidas a diferentes níveis de estresse hídrico. 1998. 105f. Dissertação (Mestrado em Engenharia Florestal) Universidade Federal do Paraná, Curitiba, 1998

SILVA, R. B. G.; SIMÕES, D.; SILVA, M. R. Qualidade de mudas clonais de Eucalyptus urophylla x E. grandis em função do substrato. 
Revista Brasileira de Engenharia Agrícola e Ambiental, v.16, n.3, p.297-302, 2012.

TELES, C. R.; COSTA, A. N.; GONCALVES, R. F. Produção de lodo de esgoto em lagoas de estabilização e o seu uso no cultivo de espécies florestais na região sudoeste do Brasil. Sanare, v.12, n.12, p.53-60, 1999.

TRIGUEIRO, R. M.; GUERRINI, I. A. Produção de mudas de pinus em substrato composto por

biossólido e casca de arroz carbonizada. Revista
Energia na Agricultura, v. 17, n.4, p.1-12, 2002.

TRIGUEIRO, R. M.; GUERRINI, I. A. Uso de biossólidos como substrato para produção de mudas de eucalipto. Scientia Forestalis, n.64, p.150-162, 2003.

VALERI, S. V.; CORRADINI, L. Fertilização em viveiro para produção de mudas de Eucalyptus e Pinus. In: GONÇALVES, J. L. M.; BENETTI, V., (Ed.) Nutrição e fertilização florestal. Piracicaba: IPEF, 2000. p.168-190. 
